\title{
Genetic variants in novel pathways influence blood pressure and cardiovascular disease risk
}

\author{
The International Consortium for Blood Pressure Genome-Wide Association Studies
}

\begin{abstract}
Blood pressure is a heritable trait ${ }^{1}$ influenced by several biological pathways and responsive to environmental stimuli. Over one billion people worldwide have hypertension $(\geq 140 \mathrm{~mm} \mathrm{Hg}$ systolic blood pressure or $\geq 90 \mathrm{~mm} \mathrm{Hg}$ diastolic blood pressure $)^{2}$. Even small increments in blood pressure are associated with an increased risk of cardiovascular events ${ }^{3}$. This genome-wide association study of systolic and diastolic blood pressure, which used a multi-stage design in 200,000 individuals of European descent, identified sixteen novel loci: six of these loci contain genes previously known or suspected to regulate blood pressure (GUCY1A3-GUCY1B3, NPR3-C5orf23, ADM, FURIN-FES, GOSR2, GNAS-EDN3); the other ten provide new clues to blood pressure physiology. A genetic risk score based on 29 genomewide significant variants was associated with hypertension, left ventricular wall thickness, stroke and coronary artery disease, but not kidney disease or kidney function. We also observed associations with blood pressure in East Asian, South Asian and African ancestry individuals. Our findings provide new insights into the genetics and biology of blood pressure, and suggest potential novel therapeutic pathways for cardiovascular disease prevention.
\end{abstract}

Genetic approaches have advanced the understanding of biological pathways underlying inter-individual variation in blood pressure. For example, studies of rare Mendelian blood pressure disorders have identified multiple defects in renal sodium handling pathways ${ }^{4}$. More recently two genome-wide association studies (GWAS), each of $>25,000$ individuals of European ancestry, identified 13 loci associated with systolic blood pressure (SBP), diastolic blood pressure (DBP) and hypertension ${ }^{5,6}$. We now report results of a new metaanalysis of GWAS data that includes staged follow-up genotyping to identify additional blood pressure loci.

Primary analyses evaluated associations between 2.5 million genotyped or imputed single nucleotide polymorphisms (SNPs) and SBP and DBP in 69,395 individuals of European ancestry from 29 studies (Supplementary Materials sections 1-3 and Supplementary Tables 1 and 2). Following GWAS meta-analysis, we conducted a three-stage validation experiment that made efficient use of available genotyping resources, to follow up top signals in up to 133,661 additional individuals of European descent (Supplementary Fig. 1 and Supplementary Materials section 4). Twenty-nine independent SNPs at 28 loci were significantly associated with SBP, DBP, or both in the meta-analysis combining discovery and follow-up data (Fig. 1, Table 1, Supplementary Figs 2, 3 and Supplementary Tables 3-5). All 29 SNPs attained association $P<5 \times 10^{-9}$, an order of magnitude beyond the standard genome-wide significance level for a single-stage experiment (Table 1).

Sixteen of these 29 associations were novel (Table 1). Two associations were near the FURIN and GOSR2 genes; prior targeted analyses of variants in these genes suggested they may be blood pressure loci ${ }^{7,8}$. At the CACNB2 locus we validated association for a previously reported $^{6}$ SNP, rs4373814, and detected a novel independent association for rs 1813353 (pairwise $r^{2}=0.015$ in HapMap CEU). Of our 13 previously reported associations ${ }^{5,6}$, only the association at PLCD3 was not supported by the current results (Supplementary Table 4). Some of the associations are in or near genes involved in pathways known to influence blood pressure (NPR3, GUCY1A3-GUCY1B3, $A D M, G N A S-E D N 3, N P P A-N P P B$ and CYP17A1; Supplementary Fig. 4). Twenty-two of the 28 loci did not contain genes that were a priori strong biological candidates.

As expected from prior blood pressure GWAS results, the effects of the novel variants on SBP and DBP were small (Fig. 1 and Table 1). For all variants, the observed directions of effects were concordant for SBP, DBP and hypertension (Fig. 1, Table 1 and Supplementary Fig. 3). Among the genes at the genome-wide significant loci, only CYP17A1, previously implicated in Mendelian congenital adrenal hyperplasia and hypertension, is known to harbour rare variants that have large effects on blood pressure?

We performed several analyses to identify potential causal alleles and mechanisms. First, we looked up the 29 genome-wide significant index SNPs and their close proxies $\left(r^{2}>0.8\right)$ among cis-acting expression SNP (eSNP) results from multiple tissues (Supplementary Materials section 5). For 13/29 index SNPs, we found an association between nearby eSNP variants and the expression levels of at least one gene transcript $\left(10^{-4}>P>10^{-51}\right.$; Supplementary Table 6). In five cases, the index blood pressure SNP and the best eSNP from a genomewide survey were identical, highlighting potential mediators of the SNP-blood pressure associations.

Second, because changes in protein sequence are a priori strong functional candidates, we sought non-synonymous coding SNPs that were in high linkage disequilibrium $\left(r^{2}>0.8\right)$ with the 29 index SNPs. We identified such SNPs at eight loci (Table 1, Supplementary Materials section 6 and Supplementary Table 7). In addition we performed analyses testing for differences in genetic effect according to body mass index (BMI) or sex, and analyses of copy number variants, pathway enrichment and metabolomic data, but we did not find any statistically significant results (Supplementary Materials sections 7-9 and Supplementary Tables 8-10).

We evaluated whether the blood pressure variants we identified in individuals of European ancestry were associated with blood pressure in individuals of East Asian $(N=29,719)$, South Asian $(N=23,977)$ and African $(N=19,775)$ ancestries (Table 1 and Supplementary Tables 11-13). We found significant associations in individuals of East Asian ancestry for SNPs at nine loci and in individuals of South Asian ancestry for SNPs at six loci; some have been reported previously (Supplementary Tables 12 and 15). The lack of significant association for individual SNPs may reflect small sample sizes, differences in allele frequencies or linkage disequilibrium patterns, imprecise imputation for some ancestries using existing reference samples, or a genuinely different underlying genetic architecture. Because of limited power to detect effects of individual variants in the smaller non-European samples, we created genetic risk scores for SBP and DBP incorporating all 29 blood pressure variants weighted according to effect sizes observed in the European samples. In each non-European ancestry group, risk scores were strongly associated with $\operatorname{SBP}\left(P=1.1 \times 10^{-40}\right.$ in East Asian, $P=2.9 \times 10^{-13}$ in South Asian, $P=9.8 \times 10^{-4}$ in African 
a

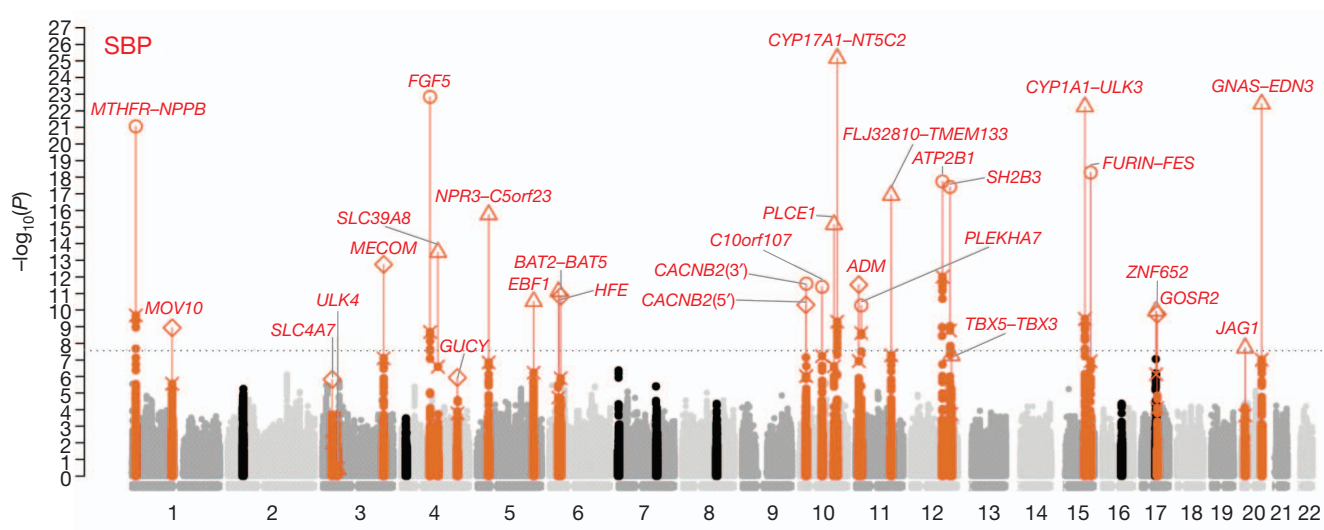

b

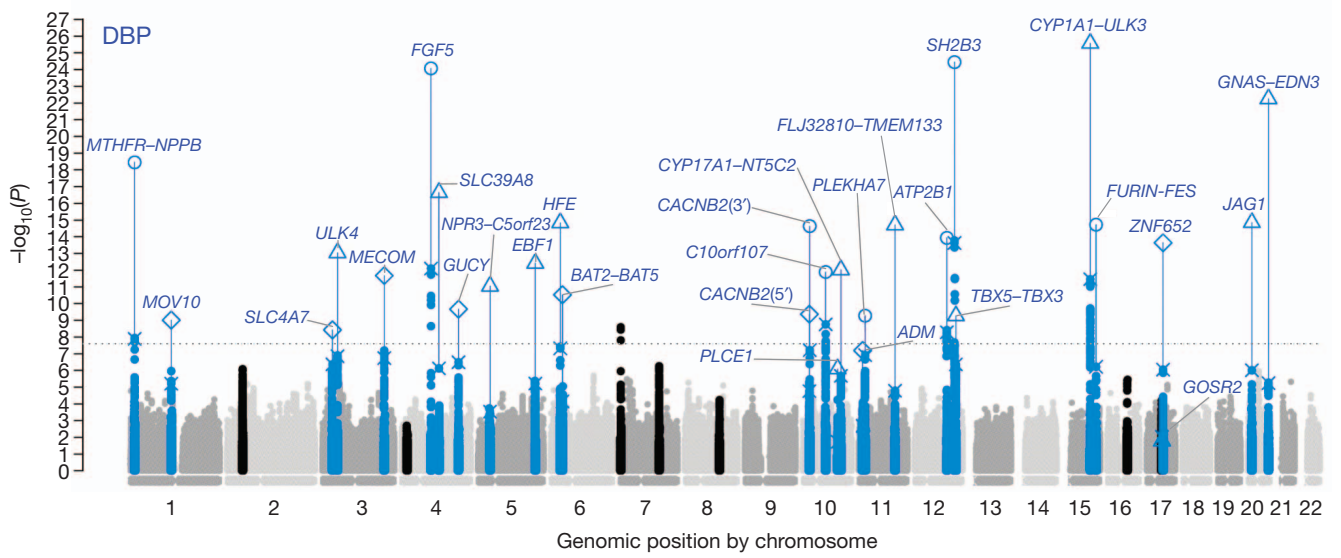

c

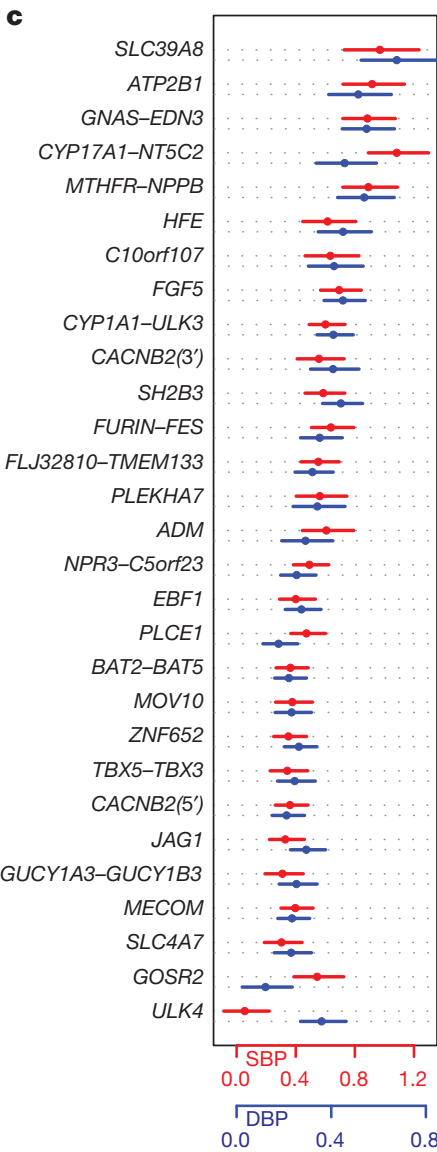

Figure $1 \mid$ Genome-wide $-\log _{10} P$-value plots and effects for significant loci. a, b, Genome-wide $-\log _{10} P$-value plots are shown for SBP (a) and DBP (b). SNPs within loci reaching genome-wide significance are labelled in red for SBP and blue for DBP ( $\pm 2.5 \mathrm{Mb}$ of lowest $P$ value) and lowest $P$ values in the initial genome-wide analysis as well as the results of analysis including validation data are labelled separately. The lowest $P$ values in the initial GWAS are denoted with a $\mathrm{X}$. The range of different sample sizes in the final meta-

ancestry individuals $)$ and $\mathrm{DBP}\left(P=2.9 \times 10^{-48}, P=9.5 \times 10^{-15}\right.$ and $P=5.3 \times 10^{-5}$, respectively; Supplementary Table 13$)$.

We also created a genetic risk score to assess association of the variants in aggregate with hypertension and with clinical measures of hypertensive complications including left ventricular mass, left ventricular wall thickness, incident heart failure, incident and prevalent stroke, prevalent coronary artery disease (CAD), kidney disease and measures of kidney function, using results from other GWAS consortia (Table 2, Supplementary Materials sections 10, 11 and Supplementary Table 14). The risk score was weighted using the average of SBP and DBP effects for the 29 SNPs. In an independent sample of 23,294 women ${ }^{10}$, an increase of one standard deviation in the genetic risk score was associated with a $23 \%$ increase in the odds of hypertension (95\% confidence interval 19-28\%; Table 2 and Supplementary Table 14). Among individuals in the top decile of the risk score, the prevalence of hypertension was $29 \%$ compared with $16 \%$ in the bottom decile (odds ratio $2.09,95 \%$ confidence interval $1.86-2.36$ ). Similar results were observed in an independent hypertension case-control sample (Table 2). In our study, individuals in the top compared to bottom quintiles of genetic risk score differed by $4.6 \mathrm{~mm} \mathrm{Hg} \mathrm{SBP}$ and $3.0 \mathrm{~mm} \mathrm{Hg} \mathrm{DBP}$, differences that approach population-averaged blood pressure treatment effects for a single antihypertensive agent ${ }^{11}$. Epidemiological data have shown that differences in SBP and DBP of this magnitude, across the population range of blood pressure, are associated with an increase in cardiovascular disease risk ${ }^{3}$. Consistent with this and in line with findings from randomized trials analysis including the validation data are indicated as: circle $(96,000-140,000)$, triangle $(>140,000-180,000)$ and diamond $(>180,000-220,000)$. SNPs near unconfirmed loci are in black. The horizontal dotted line is $P=2.5 \times 10^{-8}$. GUCY denotes GUCY1A3-GUCY1B3. c, Effect size estimates and 95\% confidence bars per blood-pressure-increasing allele of the 29 significant variants for SBP (red) and DBP (blue). Effect sizes are expressed in $\mathrm{mm} \mathrm{Hg}$ per allele.

of blood-pressure-lowering medication in hypertensive patients ${ }^{12,13}$, the genetic risk score was positively associated with left ventricular wall thickness $\left(P=6.0 \times 10^{-6}\right)$, occurrence of stroke $\left(P=3.3 \times 10^{-5}\right)$ and CAD $\left(P=8.1 \times 10^{-29}\right)$. The same genetic risk score was not, however, significantly associated with chronic kidney disease or measures of kidney function, even though these renal outcomes were available in a similar sample size as for the other outcomes (Table 2). The absence of association with kidney phenotypes could be explained by a weaker causal relationship between blood pressure and kidney phenotypes than with CAD and stroke. This finding is consistent with the mismatch between observational data that show a positive association of blood pressure with kidney disease, and clinical trial data that show inconsistent evidence of a benefit from blood pressure lowering on kidney disease prevention in patients with hypertension ${ }^{14}$. Thus, several lines of evidence converge to indicate that blood pressure elevation may in part be a consequence rather than a cause of subclinical kidney disease.

Our discovery meta-analysis (Supplementary Fig. 2) suggests an excess of modestly significant $\left(10^{-5}<P<10^{-2}\right)$ associations probably arising from common blood pressure variants of small effect. By dividing our principal GWAS data set into non-overlapping discovery $(N \approx 56,000)$ and validation $(N \approx 14,000)$ subsets, we found robust evidence for the existence of such undetected common variants (Supplementary Fig. 5 and Supplementary Materials section 12). We estimate $^{15}$ that there are 116 (95\% confidence interval 57-174) independent blood pressure variants with effect sizes similar to those 
Table 1 | Summary association results for 29 blood pressure SNPs

\begin{tabular}{|c|c|c|c|c|c|c|c|c|c|c|c|c|c|c|c|}
\hline \multirow[t]{2}{*}{ Locus } & \multirow[t]{2}{*}{ Index SNP } & \multirow[t]{2}{*}{ Chr } & \multirow[t]{2}{*}{ Position } & \multirow{2}{*}{$\begin{array}{l}\text { CA/ } \\
\text { NCA }\end{array}$} & \multirow[t]{2}{*}{ CAF } & \multirow[t]{2}{*}{ nsSNP } & \multirow[t]{2}{*}{ eSNP } & \multicolumn{3}{|c|}{ SBP } & \multicolumn{3}{|c|}{ DBP } & \multicolumn{2}{|r|}{ HTN } \\
\hline & & & & & & & & Beta & $P$ value & $\begin{array}{l}\text { Effect in } \\
\text { EA/SA/A }\end{array}$ & Beta & $P$ value & $\begin{array}{l}\text { Effect in } \\
\text { EA/SA/A }\end{array}$ & Beta & $P$ value \\
\hline MOV10 & rs2932538 & 1 & $113,018,066$ & $G / A$ & 0.75 & $Y(p)$ & $Y(p)$ & 0.388 & $1.2 \times 10^{-9}$ & $+/+/-$ & 0.240 & $9.9 \times 10^{-10}$ & $+/+* /-$ & 0.049 & $2.9 \times 10^{-7}$ \\
\hline SLCAA7 & 3082711 & 3 & $27,512,913$ & $\mathrm{~T} / \mathrm{C}$ & 0.78 & $Y(p)$ & $Y(p)$ & -0.315 & $1.5 \times 10^{-6}$ & $-1-1+$ & -0.238 & $3.8 \times 10^{-9}$ & $-1-1+$ & -0.035 & $3.6 \times 10^{-4}$ \\
\hline MECOM & 419076 & 3 & $170,583,580$ & $\mathrm{~T} / \mathrm{C}$ & 0.47 & - & - & 0.409 & $1.8 \times 10^{-13}$ & $+/+/+$ & 0.241 & $2.1 \times 10^{-12}$ & $+1+1-$ & 0.031 & $3.1 \times 10^{-4}$ \\
\hline SLC39A8 & 13107325 & 4 & $103,407,732$ & $\mathrm{~T} / \mathrm{C}$ & 0.05 & Y & $Y(+)$ & -0.981 & $3.3 \times 10^{-14}$ & $? /+/+$ & -0.684 & $2.3 \times 10^{-17}$ & $? /+/+$ & -0.105 & $4.9 \times 10^{-7}$ \\
\hline $\begin{array}{l}\text { GUCY1A3- } \\
\text { GUCY1B3 }\end{array}$ & rs13139571 & 4 & $156,864,963$ & $\mathrm{C} / \mathrm{A}$ & 0.76 & - & - & 0.321 & $1.2 \times 10^{-6}$ & $+/-1+$ & 0.260 & $2.2 \times 10^{-10}$ & $+/-1+$ & 0.042 & $2.5 \times 10^{-5}$ \\
\hline $\begin{array}{l}\text { NPR3- } \\
\text { C5orf23 }\end{array}$ & rs1173771 & 5 & $32,850,785$ & $G / A$ & 0.60 & - & - & 0.504 & $1.8 \times 10^{-16}$ & $+* /+/+$ & 0.261 & $9.1 \times 10^{-12}$ & $+* /+/-$ & 0.062 & $3.2 \times 10^{-10}$ \\
\hline EBF1 & rs11953630 & 5 & $157,777,980$ & $\mathrm{~T} / \mathrm{C}$ & 0.37 & - & - & -0.412 & $3.0 \times 10^{-11}$ & $+/+/+$ & -0.281 & $3.8 \times 10^{-13}$ & $+/+/+$ & -0.052 & $1.7 \times 10^{-7}$ \\
\hline HFE & 945 & 6 & $26,199,158$ & $\mathrm{G} / \mathrm{C}$ & 0.14 & Y & - & 0.627 & $7.7 \times 10$ & $+/+/-$ & 0.457 & $1.5 \times$ & $+1+1-$ & 0.095 & $10^{-10}$ \\
\hline AT2-BAT5 & 805303 & 6 & $31,724,345$ & $\mathrm{G} / \mathrm{A}$ & 0.61 & $Y(p)$ & $Y(+)$ & 0.376 & $1.5 \times 10^{-11}$ & $-/-/ ?$ & 0.228 & $3.0 \times 10^{-11}$ & $-/-/+$ & 0.054 & $1.1 \times 10^{-10}$ \\
\hline VB2( $\left.5^{\prime}\right)$ & & 10 & $18,459,978$ & $\mathrm{G} / \mathrm{C}$ & 0.55 & - & - & -0.373 & $4.8 \times$ & $+1+1-$ & -0.218 & $4.4 \times 10^{-10}$ & $-1+/-$ & -0.046 & \\
\hline PLCE1 & 932764 & 10 & $95,885,930$ & $\mathrm{G} / \mathrm{A}$ & 0.44 & - & - & 0.484 & $7.1 \times 10^{-16}$ & $+1+1-$ & 0.185 & $8.1 \times 10^{-7}$ & $+1+1-$ & 0.055 & $9.4 \times 10^{-9}$ \\
\hline$A D M$ & rs7129220 & 11 & $10,307,114$ & $\mathrm{G} / \mathrm{A}$ & 0.89 & - & - & -0.619 & $3.0 \times 10^{-12}$ & $? /-/+$ & -0.299 & $6.4 \times 10^{-8}$ & $? /-/+$ & -0.044 & $1.1 \times 10^{-3}$ \\
\hline $\begin{array}{l}\text { FLJ32810- } \\
\text { TMEM133 }\end{array}$ & rs633185 & 11 & $100,098,748$ & $\mathrm{G} / \mathrm{C}$ & 0.28 & - & - & -0.565 & $1.2 \times 10^{-17}$ & $+* /+/+$ & -0.328 & $2.0 \times 10^{-15}$ & $+* /+/-$ & -0.070 & $5.4 \times 10^{-11}$ \\
\hline FURIN-FES & rs2521501 & 15 & $89,238,392$ & $T / A$ & 0.31 & - & $\mathrm{Y}(-)$ & 0.650 & $5.2 \times 10^{-19}$ & $+* /+/+$ & 0.359 & $1.9 \times 10^{-15}$ & $+* /+/+$ & 0.059 & $7.0 \times 10^{-7}$ \\
\hline GOSR2 & & 17 & & T/C & 0.8 & - & $Y(+)$ & -0.5 & $1.1 \times$ & $+/-/+$ & -0 & 0. & $+/-1+$ & -0 & 0.08 \\
\hline G1 & 235 & 20 & $10,917,030$ & $\mathrm{G} / \mathrm{A}$ & 0.46 & - & - & 0.340 & $1.9 \times 10^{-8}$ & $+* /+/+$ & 0.302 & $1.4 \times 10^{-15}$ & $+* /+* /+$ & 0.034 & $4.6 \times 10^{-4}$ \\
\hline GNAS-EDN3 & rs6015450 & 20 & $57,184,512$ & $\mathrm{G} / \mathrm{A}$ & 0.12 & $Y(p)$ & - & 0.896 & $3.9 \times 10^{-23}$ & $? /+/+$ & 0.557 & $5.6 \times 10^{-23}$ & $? /+* /+$ & 0.110 & $4.2 \times 10^{-14}$ \\
\hline $\begin{array}{l}\text { MTHFR- } \\
\text { NPPB }\end{array}$ & rs17367504 & 1 & $11,785,365$ & $\mathrm{G} / \mathrm{A}$ & 0.15 & - & $Y(-/ r)$ & -0.903 & $8.7 \times 10^{-22}$ & $+/+/+$ & -0.547 & $3.5 \times 10^{-19}$ & $+/+/+$ & -0.103 & $2.3 \times 10^{-10}$ \\
\hline ULK4 & rs3774372 & 3 & $41,852,418$ & $T / C$ & 0.83 & Y & $Y(r / p)$ & -0.067 & 0.39 & $-/-1+$ & -0.367 & $9.0 \times 10^{-14}$ & $+/+/+$ & -0.017 & \\
\hline FGF5 & 1458038 & 4 & 3,747 & T/C & 0.29 & - & - & 0.706 & $1.5 \times 10^{-23}$ & $+* /+/+$ & 0.457 & $8.5 \times 10^{-25}$ & $+* /+* /+$ & 0.072 & $1.9 \times 10^{-7}$ \\
\hline CACNB2(3') & rs1813353 & 10 & $18,747,454$ & $\mathrm{~T} / \mathrm{C}$ & 0.68 & - & - & 0.569 & $2.6 \times 10^{-12}$ & $+/+/+$ & 0.415 & $2.3 \times 10^{-15}$ & $+/+/+$ & 0.078 & $6.2 \times 10^{-10}$ \\
\hline C10orf107 & & 10 & 7,559 & $\mathrm{G} / \mathrm{C}$ & 0.8 & - & $Y(r)$ & 0.646 & $4.0 \times 10^{-12}$ & $-/+/-$ & 0.4 & $1.3 \times$ & $-/-/-$ & 0.096 & $\times 10^{-9}$ \\
\hline $\begin{array}{l}\text { CYP17A1- } \\
\text { NT5C2 }\end{array}$ & rs11191548 & 10 & $104,836,168$ & $\mathrm{~T} / \mathrm{C}$ & 0.91 & - & $Y(-)$ & 1.095 & $6.9 \times 10^{-26}$ & $+* /+* /+$ & 0.464 & $9.4 \times 10^{-13}$ & $+* /+* /+$ & 0.097 & $1.4 \times 10^{-5}$ \\
\hline PLEKHA7 & rs 38 & 11 & & $\mathrm{~T} / \mathrm{C}$ & 0.26 & - & - & 0.575 & $5.3 \times 10^{-11}$ & $+* /+/+$ & & $10^{-10}$ & $+* /-/+$ & 0.062 & $3.4 \times 10^{-6}$ \\
\hline ATP2B1 & 17249754 & 12 & $88,584,717$ & $\mathrm{G} / \mathrm{A}$ & 0.84 & - & - & & $1.8 \times 10^{-18}$ & $+* /+* /-$ & & $1.2 \times 10^{-14}$ & $+* /+* /-$ & 0.126 & $1.1 \times 10^{-14}$ \\
\hline SH2B3 & rs3184504 & 12 & $110,368,991$ & $\mathrm{~T} / \mathrm{C}$ & 0.47 & Y & $Y(+)$ & 0.598 & $3.8 \times 10^{-18}$ & $-/-/+$ & 0.448 & $3.6 \times 10^{-25}$ & $-/-/+$ & 0.056 & $2.6 \times 10^{-6}$ \\
\hline TBX5-TBX3 & rs10850411 & 12 & $113,872,179$ & $T / C$ & 0.7 & - & - & & $5.4 \times 10^{-8}$ & $-/+/-$ & & $5.4 \times 10^{-10}$ & $-/-1-$ & 0.045 & \\
\hline $\begin{array}{l}\text { CYP1A1- } \\
\text { ULK3 }\end{array}$ & rs1378942 & 15 & $72,864,420$ & $\mathrm{C} / \mathrm{A}$ & 0.35 & - & $Y(+)$ & 0.613 & $5.7 \times 10^{-23}$ & $+* /+/+$ & 0.416 & $2.7 \times 10^{-26}$ & $+* /+/-$ & 0.073 & $1.0 \times 10^{-8}$ \\
\hline ZNF652 & rs12940887 & 17 & $44,757,806$ & $\mathrm{~T} / \mathrm{C}$ & 0.38 & - & $Y(-)$ & 0.362 & $1.8 \times 10^{-10}$ & $+/-1+$ & 0.27 & $2.3 \times 10^{-14}$ & $+/-/+$ & 0.046 & $1.2 \times 10^{-7}$ \\
\hline
\end{tabular}

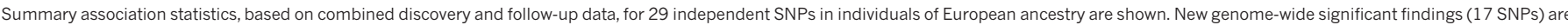

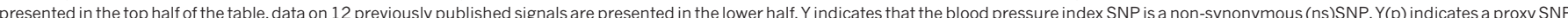

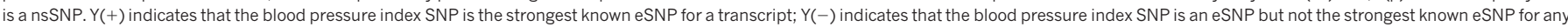

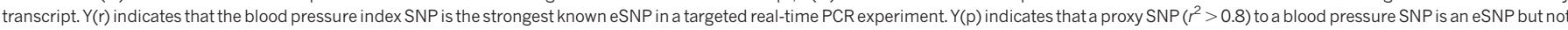

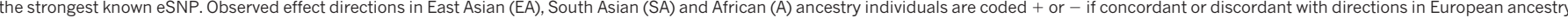

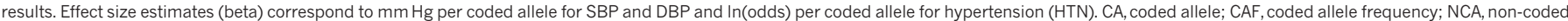
allele. ? denotes missing data. Genomic positions use NCBI Build 36 coordinates.

* Significant, controlling the FDR at $5 \%$ over 58 tests per ancestry (Supplementary Tables 5 and 12).

reported here, which collectively can explain $\sim 2.2 \%$ of the phenotypic variance for SBP and DBP, compared with $0.9 \%$ explained by the 29 associations discovered thus far (Supplementary Fig. 6 and Supplementary Materials section 13).

Most of the 28 blood pressure loci harbour multiple genes (Supplementary Table 15 and Supplementary Fig. 4), and although substantial research is required to identify the specific genes and variants responsible for these associations, several loci contain highly plausible biological candidates. The NPPA and $N P P B$ genes at the $M T H F R-N P P B$ locus encode precursors for atrial- and B-type natriuretic peptides (ANP, BNP), and previous work has identified SNPs - modestly correlated with our index SNP at this locus-which are associated with plasma ANP, BNP and blood pressure ${ }^{16}$. We found the index SNP at this locus was associated with opposite effects on blood pressure and on ANP/BNP levels, consistent with a model in which the variants act through increased ANP/BNP production to lower blood pressure ${ }^{16}$ (Supplementary Materials section 14).

Two other loci identified in the current study harbour genes involved in natriuretic peptide and related nitric oxide signalling pathways $^{17,18}$, both of which act to regulate cyclic guanosine monophosphate. The first locus contains NPR3, which encodes the natriuretic peptide clearance receptor (NPR-C). NPR3 knockout mice exhibit reduced clearance of circulating natriuretic peptides and lower blood pressure $^{19}$. The second locus includes GUCY1A3 and GUCY1B3, encoding the $\alpha$ and $\beta$ subunits of soluble guanylate cyclase; knockout of either gene in murine models results in hypertension ${ }^{20}$.
Another locus contains $A D M$-encoding adrenomedullin-which has natriuretic, vasodilatory and blood-pressure-lowering properties ${ }^{21}$. At the GNAS-EDN3 locus, ZNF831 is closest to the index SNP, but GNAS and EDN3 are two nearby compelling biological candidates (Supplementary Fig. 4 and Supplementary Table 15).

We identified two loci with plausible connections to blood pressure via genes implicated in renal physiology or kidney disease. At the first locus, SLC4A7 is an electro-neutral sodium bicarbonate co-transporter expressed in the nephron and in vascular smooth muscle ${ }^{22}$. At the second locus, PLCE1 (phospholipase-C-epsilon-1 isoform) is important for normal podocyte development in the glomerulus; sequence variation in PLCE1 has been implicated in familial nephrotic syndromes and end-stage kidney disease ${ }^{23}$.

Missense variants in two genes involved in metal ion transport were associated with blood pressure in our study. The first encodes a His/ Asp change at amino acid 63 (H63D) in HFE and is a low-penetrance allele for hereditary hemochromatosis ${ }^{24}$. The second is an Ala/Thr polymorphism located in exon 7 of SLC39A8, which encodes a zinc transporter that also transports cadmium and manganese ${ }^{25}$. The same allele of SLC39A8 associated with blood pressure in our study has recently been associated with high-density lipoprotein cholesterol levels ${ }^{26}$ and $\mathrm{BMI}^{27}$ (Supplementary Table 15).

We have shown that 29 independent genetic variants influence blood pressure in people of European ancestry. The variants reside in 28 loci, 16 of which were novel, and we confirmed association of several of them in individuals of non-European ancestry. A risk score 
Table 2 | Genetic risk score and cardiovascular outcome association results

\begin{tabular}{|c|c|c|c|c|c|c|c|c|c|c|}
\hline \multirow[t]{2}{*}{ Phenotype } & \multirow[t]{2}{*}{ Source } & Effect & \multicolumn{2}{|l|}{ s.e. } & \multirow[t]{2}{*}{$P$ value } & \multirow[t]{2}{*}{ No. SNPs } & \multicolumn{3}{|c|}{ Contrast top versus bottom } & \multirow{2}{*}{$\begin{array}{c}N \text { case/control } \\
\text { or total }\end{array}$} \\
\hline & & \multicolumn{3}{|c|}{ (per s.d. of genetic risk score) } & & & Quintiles & \multicolumn{2}{|l|}{ Deciles } & \\
\hline \multicolumn{11}{|l|}{ Blood pressure phenotypes } \\
\hline $\begin{array}{l}\text { SBP }(\mathrm{mm} \mathrm{Hg}) \\
\text { DBP }(\mathrm{mm} \mathrm{Hg}) \\
\text { Prevalent hypertension } \\
\text { Prevalent hypertension }\end{array}$ & $\begin{array}{l}\text { WGHS } \\
\text { WGHS } \\
\text { WGHS } \\
\text { BRIGHT }\end{array}$ & $\begin{array}{l}1.645 \\
1.057 \\
0.211 \\
0.287\end{array}$ & $\begin{array}{l}0.098 \\
0.067 \\
0.018 \\
0.031\end{array}$ & $\begin{array}{l}\text { (a) } \\
\text { (a) } \\
\text { (b) } \\
\text { (b) }\end{array}$ & $\begin{array}{l}6.5 \times 10^{-63} \\
8.4 \times 10^{-57} \\
3.1 \times 10^{-33} \\
7.7 \times 10^{-21}\end{array}$ & $\begin{array}{l}29 \\
29 \\
29 \\
29\end{array}$ & $\begin{array}{l}4.61 \\
2.96 \\
1.80 \\
2.23\end{array}$ & $\begin{array}{l}5.77 \\
3.71 \\
2.09 \\
2.74\end{array}$ & $\begin{array}{l}\text { (a) } \\
\text { (a) } \\
\text { (b) } \\
\text { (b) }\end{array}$ & $\begin{array}{r}23,294 \\
23,294 \\
5,018 / 18,276 \\
2,406 / 1,990\end{array}$ \\
\hline \multicolumn{11}{|l|}{ Dichotomous endpoints } \\
\hline $\begin{array}{l}\text { Incident heart failure } \\
\text { Incident stroke } \\
\text { Prevalent stroke } \\
\text { Stroke (combined, incident and prevalent) } \\
\text { Prevalent CAD } \\
\text { Prevalent CAD } \\
\text { Prevalent CAD } \\
\text { Prevalent CAD (combined) } \\
\text { Prevalent chronic kidney disease } \\
\text { Prevalent microalbuminuria }\end{array}$ & $\begin{array}{l}\text { CHARGE-HF } \\
\text { NEURO-CHARGE } \\
\text { SCG } \\
\text { CHARGE \& SCG } \\
\text { CARDIoGRAM } \\
\text { C4D ProCARDIS } \\
\text { C4D HPS } \\
\text { CARDIoGRAM \& C4D } \\
\text { CKDGen } \\
\text { CKDGen }\end{array}$ & $\begin{array}{l}0.035 \\
0.103 \\
0.075 \\
\text { NA } \\
0.092 \\
0.132 \\
0.083 \\
0.100 \\
0.014 \\
0.008\end{array}$ & $\begin{array}{r}0.021 \\
0.028 \\
0.037 \\
N A \\
0.010 \\
0.022 \\
0.027 \\
0.009 \\
0.015 \\
0.019\end{array}$ & $\begin{array}{l}\text { (c) } \\
\text { (c) } \\
\text { (b) } \\
\text { NA } \\
\text { (b) } \\
\text { (b) } \\
\text { (b) } \\
\text { (b) } \\
\text { (b) } \\
\text { (b) }\end{array}$ & $\begin{array}{l}0.10 \\
0.0002 \\
0.05 \\
3.3 \times 10^{-5} \\
1.6 \times 10^{-19} \\
2.2 \times 10^{-9} \\
0.002 \\
8.1 \times 10^{-29} \\
0.35 \\
0.68\end{array}$ & $\begin{array}{l}29 \\
28 \\
29 \\
\text { NA } \\
28 \\
29 \\
29 \\
29 \\
29 \\
29\end{array}$ & $\begin{array}{l}1.10 \\
1.34 \\
1.23 \\
\text { NA } \\
1.29 \\
1.45 \\
1.26 \\
1.32 \\
1.04 \\
1.02\end{array}$ & $\begin{array}{l}1.13 \\
1.44 \\
1.30 \\
\text { NA } \\
1.38 \\
1.59 \\
1.34 \\
1.42 \\
1.05 \\
1.03\end{array}$ & $\begin{array}{l}\text { (c) } \\
\text { (c) } \\
\text { (b) } \\
\text { NA } \\
\text { (b) } \\
\text { (b) } \\
\text { (b) } \\
\text { (b) } \\
\text { (b) } \\
\text { (b) }\end{array}$ & $\begin{array}{r}2,526 / 18,400 \\
1,544 / 18,058 \\
1,473 / 1,482 \\
3,017 / 19,540 \\
22,233 / 64,726 \\
5,720 / 4,381 \\
2,704 / 2,804 \\
30,657 / 71,911 \\
5,807 / 61,286 \\
3,698 / 27,882\end{array}$ \\
\hline \multicolumn{11}{|l|}{ Continuous measures of target organ damage } \\
\hline $\begin{array}{l}\text { Left ventricular mass }(\mathrm{g}) \\
\text { Left ventricular wall thickness (cm) } \\
\text { Serum creatinine } \\
\text { eGFR (four-parameter MDRD equation) } \\
\text { Urinary albumin/creatinine ratio }\end{array}$ & $\begin{array}{l}\text { EchoGen } \\
\text { EchoGen } \\
\text { KidneyGen } \\
\text { CKDGen } \\
\text { CKDGen }\end{array}$ & $\begin{array}{c}0.822 \\
0.009 \\
-0.001 \\
-0.0001 \\
0.005\end{array}$ & $\begin{array}{l}0.317 \\
0.002 \\
0.001 \\
0.0009 \\
0.007\end{array}$ & $\begin{array}{l}\text { (a) } \\
\text { (a) } \\
\text { (d) } \\
\text { (d) } \\
\text { (d) }\end{array}$ & $\begin{array}{l}0.01 \\
6.0 \times 10^{-6} \\
0.24 \\
0.93 \\
0.43\end{array}$ & $\begin{array}{l}29 \\
29 \\
29 \\
29 \\
29\end{array}$ & $\begin{array}{l}2.30 \\
0.03 \\
1.00 \\
1.00 \\
1.01\end{array}$ & $\begin{array}{l}2.89 \\
0.03 \\
1.00 \\
1.00 \\
1.02\end{array}$ & $\begin{array}{l}\text { (a) } \\
\text { (a) } \\
\text { (d) } \\
\text { (d) } \\
\text { (d) }\end{array}$ & $\begin{array}{l}12,612 \\
12,612 \\
23,812 \\
67,093 \\
31,580\end{array}$ \\
\hline
\end{tabular}

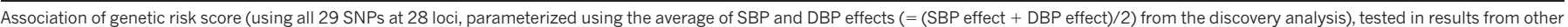

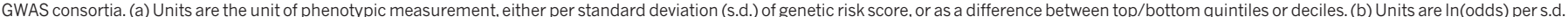

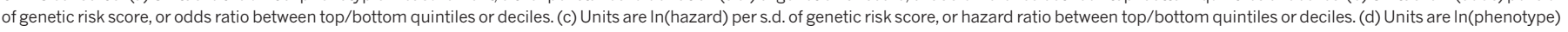

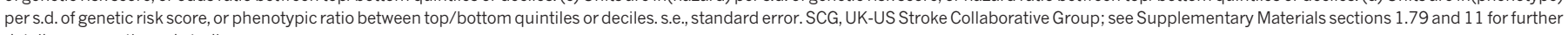
detail on consortia and studies.

derived from the 29 variants was significantly associated with bloodpressure-related organ damage and clinical cardiovascular disease, but not kidney disease. These loci improve our understanding of the genetic architecture of blood pressure, provide new biological insights into blood pressure control and may identify novel targets for the treatment of hypertension and the prevention of cardiovascular disease.

Note added in proof: Since this manuscript was submitted, Kato et al. published a blood pressure GWAS in East Asians that identified a SNP highly correlated to the SNP we report at the NPR3/C5orf23 locus ${ }^{28}$.

\section{METHODS SUMMARY}

Supplementary Materials provide complete methods and include the following sections: study recruitment and phenotyping, adjustment for antihypertensive medications, genotyping, data quality control, genotype imputation, withincohort association analyses, meta-analyses of discovery and validation stages, stratified analyses by sex and BMI, identification of eSNPs and non-synonymous SNPs, metabolomic and lipidomic analyses, CNV analyses, pathway analyses, analyses for non-European ancestries, association of a risk score with hypertension and cardiovascular disease, estimation of numbers of undiscovered variants, measurement of natriuretic peptides, and brief literature reviews and GWAS database lookups of all validated blood pressure loci. Full GWAS results for $\approx 2.5$ million SNPs are also provided.

Received 16 August 2010; accepted 28 July 2011.

Published online 11 September 2011.

1. Levy, D. et al. Evidence for a gene influencing blood pressure on chromosome 17. Genome scan linkage results for longitudinal blood pressure phenotypes in subjects from the Framingham heart study. Hypertension 36, 477-483 (2000).

2. Kearney, P. M. et al. Global burden of hypertension: analysis of worldwide data. Lancet 365, 217-223 (2005).

3. Prospective Studies Collaboration. Age-specific relevance of usual blood pressure to vascular mortality: a meta-analysis of individual data for one million adults in 61 prospective studies. Lancet 360, 1903-1913 (2002).

4. Lifton, R. P., Gharavi, A. G. \& Geller, D. S. Molecular mechanisms of human hypertension. Cell 104, 545-556 (2001)

5. Newton-Cheh, C. et al. Genome-wide association study identifies eight loci associated with blood pressure. Nature Genet. 41, 666-676 (2009).

6. Levy, D. et al. Genome-wide association study of blood pressure and hypertension Nature Genet. 41, 677-687 (2009).
7. Meyer, T. E. etal. GOSR2 Lys67Arg is associated with hypertension in whites. Am. J. Hypertens. 22, 163-168 (2009).

8. Li, N. et al. Associations between genetic variations in the FURIN gene and hypertension. BMC Med. Genet. 11, 124 (2010).

9. Mussig, K. et al. 17 $\alpha$-hydroxylase/17,20-lyase deficiency caused by a novel homozygous mutation (Y27Stop) in the cytochrome CYP17 gene. J. Clin. Endocrinol. Metab. 90, 4362-4365 (2005).

10. Ridker, P. M. et al. Rationale, design, and methodology of the Women's Genome Health Study: a genome-wide association study of more than 25,000 initially healthy american women. Clin. Chem. 54, 249-255 (2008).

11. Burt, V. L. et al. Trends in the prevalence, awareness, treatment, and control of hypertension in the adult US population. Data from the health examination surveys, 1960 to 1991 . Hypertension 26, 60-69 (1995).

12. Blood Pressure Lowering Treatment Trialists' Collaboration Effects of different regimens to lower blood pressure on major cardiovascular events in older and younger adults: meta-analysis of randomised trials. Br. Med. J. 336, 1121-1123 (2008)

13. Law, M. R., Morris, J. K. \& Wald, N. J. Use of blood pressure lowering drugs in the prevention of cardiovascular disease: meta-analysis of 147 randomised trials in the context of expectations from prospective epidemiological studies. Br. Med. J. 338, b1665 (2009).

14. Lewis, J. B. Blood pressure control in chronic kidney disease: is less really more? J. Am. Soc. Nephrol. 21, 1086-1092 (2010).

15. Park, J. H. etal. Estimation of effect size distribution from genome-wide association studies and implications for future discoveries. Nature Genet. 42, 570-575 (2010).

16. Newton-Cheh, C. et al. Association of common variants in NPPA and NPPB with circulating natriuretic peptides and blood pressure. Nature Genet. 41, 348-353 (2009).

17. Schenk, D. B. et al. Purification and subunit composition of atrial natriuretic peptide receptor. Proc. Natl Acad. Sci. USA 84, 1521-1525 (1987).

18. Schmidt, H. H. \& Walter, U. NO at work. Cell 78, 919-925 (1994).

19. Matsukawa, N. et al. The natriuretic peptide clearance receptor locally modulates the physiological effects of the natriuretic peptide system. Proc. Natl Acad. Sci. USA 96, 7403-7408 (1999)

20. Friebe, A., Mergia, E., Dangel, O., Lange, A. \& Koesling, D. Fatal gastrointestinal obstruction and hypertension in mice lacking nitric oxide-sensitive guanylyl cyclase. Proc. Natl Acad. Sci. USA 104, 7699-7704 (2007).

21. Ishimitsu, T., Ono, H., Minami, J. \& Matsuoka, H. Pathophysiologic and therapeutic implications of adrenomedullin in cardiovascular disorders. Pharmacol. Ther. 111, 909-927 (2006).

22. Pushkin, A. et al. Cloning, tissue distribution, genomic organization, and functional characterization of NBC3, a new member of the sodium bicarbonate cotransporter family. J. Biol. Chem. 274, 16569-16575 (1999).

23. Hinkes, B. et al. Positional cloning uncovers mutations in PLCE1 responsible for a nephrotic syndrome variant that may be reversible. Nature Genet. 38, 1397-1405 (2006). 
24. Feder, J. N. et al. A novel MHC class I-like gene is mutated in patients with hereditary haemochromatosis. Nature Genet. 13, 399-408 (1996).

25. He, L., Wang, B., Hay, E. B. \& Nebert, D. W. Discovery of ZIP transporters that participate in cadmium damage to testis and kidney. Toxicol. Appl. Pharmacol. 238, 250-257 (2009).

26. Teslovich, T. M. et al. Biological, clinical and population relevance of 95 loci for blood lipids. Nature 466, 707-713 (2010).

27. Speliotes, E. K. et al. Association analyses of 249,796 individuals reveal 18 new loci associated with body mass index. Nature Genet. 42, 937-948 (2010)

28. Kato, N. et al. Meta-analysis of genome-wide association studies identifies common variants associated with blood pressure variation in east Asians. Nature Genet. 43, 531-538 (2011).

Supplementary Information is linked to the online version of the paper at www.nature.com/nature.

Acknowledgements A number of the participating studies and authors are members of the CHARGE and Global BPgen consortia. Many funding mechanisms by NIH/ NHLBI, European and private funding agencies contributed to this work and a full list is provided in section 21 of the Supplementary Materials.

Author Contributions Full author contributions and roles are listed in Supplementary Materials section 19

Author Information Reprints and permissions information is available at www.nature.com/reprints. The authors declare no competing financial interests. Readers are welcome to comment on the online version of this article at www.nature.com/nature. Correspondence and requests for materials should be addressed to A.C. (aravinda@jhmi.edu), M.C. (m.j.caulfield@qmul.ac.uk), D.L. (levyd@nhlbi.nih.gov),P.B.M. (p.b.munroe@qmul.ac.uk), C.N.-C. (cnewtoncheh@chgr.mgh.harvard.edu).

Georg B. Ehrett,2,3*, Patricia B. Munroe ${ }^{4 *}$, Kenneth M. Rice ${ }^{5 *}$, Murielle Bochud ${ }^{2 *}$, Andrew D. Johnson ${ }^{6,7 *}$, Daniel I. Chasman ${ }^{8,9 *}$, Albert V. Smith ${ }^{10,11 *}$, Martin D. Tobin ${ }^{12}$ Germaine C. Verwoert ${ }^{13,14,15}$, Shih-Jen Hwang ${ }^{6,7,16}$, Vasyl Pihur ${ }^{1}$, Peter Vollenweider ${ }^{17}$, Paul F. O'Reilly ${ }^{18}$, Najaf Amin ${ }^{13}$, Jennifer L. Bragg-Gresham ${ }^{19}$, Alexander Teumer ${ }^{20}$, Nicole L. Glazer ${ }^{21}$, Lenore Launer ${ }^{22}$, Jing Hua Zhao ${ }^{23}$, Yurii Aulchenko ${ }^{13}$, Simon Heath ${ }^{24}$, Siim Sõber ${ }^{25}$, Afshin Parsa ${ }^{26}$, Jian'an Luan ${ }^{23}$, Pankaj Arora $^{27}$, Abbas Dehghan ${ }^{13,14,15}$, Feng Zhang ${ }^{28}$, Gavin Lucas ${ }^{29}$, Andrew A. Hicks ${ }^{30}$, Anne U. Jackson ${ }^{31}$, John F Peden ${ }^{32}$, Toshiko Tanaka ${ }^{33}$, Sarah H. Wild ${ }^{34}$, Igor Rudan $^{35,36}$, Wilmar $/$ II $^{37}$, Yuri Milaneschi ${ }^{33}$, Alex N. Parker ${ }^{38}$, Cristiano Fava ${ }^{39,40}$, John C. Chambers ${ }^{18,41}$, Ervin R. Fox ${ }^{42}$, Meena Kumari ${ }^{43}$, Min Jin Go ${ }^{44}$, Pim van der Harst ${ }^{45}$ Wen Hong Linda Kao ${ }^{46}$, Marketa Sjögren ${ }^{39}$, D. G. Vinay ${ }^{47}$, Myriam Alexander ${ }^{48}$, Yasuharu Tabara ${ }^{49}$, Sue Shaw-Hawkins ${ }^{4}$, Peter H. Whincup ${ }^{50}$, Yongmei Liu ${ }^{51}$, 'Gang $\mathrm{Shi}^{52}$, Johanna Kuusisto ${ }^{53}$, Bamidele Tayo ${ }^{54}$, Mark Seielstad ${ }^{55,56}$, Xueling Sim ${ }^{57}$ Khanh-Dung Hoang Nguyen ${ }^{1}$, Terho Lehtimäki ${ }^{58}$, Giuseppe Matullo ${ }^{59,60}$, Ying Wu ${ }^{61}$ Tom R. Gaunt ${ }^{62}$, N. Charlotte Onland-Moret ${ }^{63,64}$, Matthew N. Cooper ${ }^{65}$, Carl G. P. Platou ${ }^{66}$, Elin Org ${ }^{25}$, Rebecca Hardy ${ }^{67}$, Santosh Dahgam ${ }^{68}$, Jutta Palmen ${ }^{69}$, Veronique Vitart $^{70}$, Peter S. Braund ${ }^{71,72}$, Tatiana Kuznetsova ${ }^{73}$, Cuno S. P. M. Uiterwaal ${ }^{63}$, Adebowale Adeyemo $^{74}$, Walter Palmas ${ }^{75}$, Harry Campbell ${ }^{35}$, Barbara Ludwig ${ }^{76}$, Maciej Tomaszewski ${ }^{71,72}$, loanna Tzoulaki $i^{77,78}$, Nicholette D. Palmer ${ }^{79}$, CARDIoGRAM consortium†, CKDGen Consortium†, KidneyGen Consortium†, EchoGen consortium† CHARGE-HF consortium $\uparrow$, Thor Aspelund ${ }^{10,11}$, Melissa Garcia ${ }^{22}$, Yen-Pei C. Chang ${ }^{26}$, Jeffrey R. O'Connel| ${ }^{26}$, Nanette I. Steinle ${ }^{26}$, Diederick E. Grobbee ${ }^{63}$, Dan E. Arking ${ }^{1}$ Sharon L. Kardia ${ }^{80}$, Alanna C. Morrison ${ }^{81}$, Dena Hernandez ${ }^{82}$, Samer Najjar ${ }^{83,84}$, Wendy L. McArdle ${ }^{85}$, David Hadley ${ }^{50,86}$, Morris J. Brown ${ }^{87}$, John M. Connell ${ }^{88}$, Aroon D. Hingorani ${ }^{89}$, Ian N.M. Day ${ }^{62}$, Debbie A. Lawlor ${ }^{62}$, John P. Beilby ${ }^{90,91}$, Robert W. Lawrence $^{65}$, Robert Clarke ${ }^{92}$, Jemma C. Hopewell ${ }^{92}$, Halit Ongen ${ }^{32}$, Albert W. Dreisbach $^{42}$, Yali Li93, J. Hunter Young ${ }^{94}$, Joshua C. Bis ${ }^{21}$, Mika Kähönen ${ }^{95}$, Jorma Viikari $^{96}$, Linda S. Adair ${ }^{97}$, Nanette R. Lee ${ }^{98}$, Ming-Huei Chen ${ }^{99}$, Matthias Olden ${ }^{100,101}$ Cristian Pattaro ${ }^{30}$, Judith A. Hoffman Bolton ${ }^{102}$, Anna Köttgen ${ }^{102,103}$, Sven Bergmann ${ }^{104,105}$, Vincent Mooser ${ }^{106}$, Nish Chaturvedi ${ }^{107}$, Timothy M. Frayling ${ }^{108}$ Muhammad Islam ${ }^{109}$, Tazeen H. Jafar ${ }^{109}$, Jeanette Erdmann ${ }^{110}$, Smita R. Kulkarni ${ }^{111}$ Stefan R. Bornstein ${ }^{76}$ ' Jürgen Grässler ${ }^{76}$, 'Leif Groop ${ }^{112,113}$, Benjamin F. Voight ${ }^{114}$ Johannes Kettunen ${ }^{115,116}$, Philip Howard ${ }^{117}$, Andrew Taylor ${ }^{43}$, Simonetta Guarrera ${ }^{60}$, Fulvio Ricceri ${ }^{59,60}$, Valur Emilsson ${ }^{118}$, Andrew Plump ${ }^{118}$, Inês Barroso ${ }^{119,120}$, Kay-Tee Khaw $^{48}$, Alan B. Weder ${ }^{121}$, Steven C. Hunt ${ }^{122}$, Yan V. Sun ${ }^{80}$, Richard N. Bergman ${ }^{123}$ Francis S. Collins ${ }^{124}$, Lori L. Bonnycastle ${ }^{124}$, Laura J. Scott ${ }^{31}$, Heather M. Stringham ${ }^{31}$ Leena Peltonen ${ }^{116,119,125,126}$ *, Markus Perola ${ }^{125}$, Erkki Vartiainen ${ }^{125}$, Stefan-Martin Brand $^{127,128}$, Jan A. Staessen ${ }^{3 / 3}$, Thomas J. Wang ${ }^{6,129}$, Paul R. Burton ${ }^{12,72}$, Maria Soler Artigas $^{12}$, Yanbin Dong ${ }^{130}$, Harold Snieder ${ }^{130,131}$, Xiaoling Wang ${ }^{130}$, Haidong Zhu ${ }^{130}$ Kurt K. Lohman 132 , Megan E. Rudock ${ }^{51}$, Susan R. Heckbert ${ }^{133,134}$, Nicholas L. Smith ${ }^{133,134,135}$, Kerri L. Wiggins ${ }^{136}$, Ayo Doumatey ${ }^{74}$, Daniel Shriner ${ }^{74}$, Gudrun Veldre $^{25,137}$, Margus Viigimaa ${ }^{138,139}$, Sanjay Kinra ${ }^{140}$, Dorairaj Prabhakaran ${ }^{141}$, Vika Tripathy $^{141}$, Carl D. Langefeld ${ }^{79}$, Annika Rosengren ${ }^{142}$, Dag S. Thelle ${ }^{143}$, Anna Maria Corsi $^{144}$, Andrew Singleton ${ }^{82}$, Terrence Forrester ${ }^{145}$, Gina Hilton ${ }^{1}$, Colin A. McKenzieies Tunde Salako $^{146}$, Naoharu Iwai ${ }^{147}$, Yoshikuni Kita ${ }^{148}$, Toshio Ogihara $^{149}$, Takayoshi Ohkubo ${ }^{148,150}$, Tomonori Okamura ${ }^{147,148}$, Hirotsugu Ueshima ${ }^{148,151}$, Satoshi Umemura ${ }^{152}$, Susana Eyheramendy ${ }^{153}$,'Thomas Meitinger ${ }^{154,155}$, H.-Erich Wichmann ${ }^{156,157,158}$, Yoon Shin Cho ${ }^{44}$, Hyung-Lae Kim ${ }^{44}$ Jong-Young Lee ${ }^{44}$, James Scott ${ }^{159}$, Joban S. Sehmi ${ }^{41,159}$, Weihua Zhang ${ }^{18}$, Bo Hedblad $^{39}$, Peter Nilsson ${ }^{39}$, George Davey Smith ${ }^{62}$, Andrew Wong ${ }^{67}$, Narisu Narisu ${ }^{124}$ Alena Stančáková ${ }^{53}$, Leslie J. Raffel ${ }^{160}$, Jie Yao ${ }^{160}$, Sekar Kathiresan ${ }^{27,161}$, Christopher J. O'Donnel| ${ }^{9,27,162}$, Stephen M. Schwartz ${ }^{133}$, M. Arfan Ikram ${ }^{13,15}$, W. T. Longstreth $\mathrm{Jr}^{163}$, Thomas H. Mosley $^{164}$, Sudha Seshadri ${ }^{165}$, Nick R.G. Shrine ${ }^{12}$, Louise V. Wain ${ }^{12}$,
Mario A. Morken ${ }^{124}$, Amy J. Swift ${ }^{124}$, Jaana Laitinen ${ }^{166}$, Inga Prokopenko ${ }^{51,167}$, Paavo Zitting $^{168}$, Jackie A. Cooper ${ }^{69}$, Steve E. Humphries ${ }^{69}$, John Danesh ${ }^{48}$, Asif Rasheed ${ }^{169}$ Anuj Goel ${ }^{32}$, Anders Hamsten ${ }^{170}$, Hugh Watkins ${ }^{32}$, Stephan J. L. Bakker ${ }^{171}$, Wiek H. van Gilst ${ }^{45}$, Charles S. Janipalli ${ }^{47}$, K. Radha Mani ${ }^{47}$, Chittaranjan S. Yajnik ${ }^{111}$, Albert Hofman ${ }^{13}$, Francesco U. S. Mattace-Raso $0^{13,14}$, Ben A. Oostra ${ }^{172}$, Ayse Demirkan ${ }^{13}$, Aaron Isaacs ${ }^{13}$, Fernando Rivadeneira 13,14 , Edward G. Lakatta ${ }^{173}$, Marco Orru ${ }^{174,175}$ Angelo Scuteri ${ }^{173}$, Mika Ala-Korpela ${ }^{176,177,178}$, Antti J. Kangas ${ }^{176}$, Leo-Pekka Lyytikäinen ${ }^{58}$, Pasi Soininen ${ }^{176,177}$, Taru Tukiainen ${ }^{176,179,180}$, Peter Würtz ${ }^{18,176,179}$, Rick Twee-Hee Ong ${ }^{56,57,181}$, Marcus Dörr ${ }^{182}$, Heyo K. Kroemer ${ }^{\text {'83, }}$, Uwe Völker ${ }^{20}$, Henry Völzke ${ }^{184}$, Pilar Galan ${ }^{185}$, Serge Hercberg ${ }^{185}$, Mark Lathrop ${ }^{24}$, Diana Zelenika ${ }^{24}$, Panos Deloukas $^{119}$, Massimo Mangino ${ }^{28}$, Tim D. Spector ${ }^{28}$, Guangju Zhai ${ }^{28}$, James F. Meschia $^{186}$, Michael A. Nalls ${ }^{82}$, Pankaj Sharma ${ }^{187}$, Janos Terzic ${ }^{188}$, M. V. Kranthi Kumar $^{47}$, Matthew Denniff ${ }^{71}$, Ewa Zukowska-Szczechowska ${ }^{189}$, Lynne E. Wagenknecht ${ }^{79}$, F. Gerald R. Fowkes ${ }^{190}$, Fadi J. Charchar ${ }^{191}$, Peter E. H. Schwarz ${ }^{192}$, Caroline Hayward ${ }^{70}$, Xiuqing Guo ${ }^{160}$, Charles Rotimi ${ }^{74}$, Michiel L. Bots ${ }^{63}$, Eva Brand $^{193}$, Nilesh J. Samani ${ }^{11,72}$, Ozren Polasek 194 , Philippa J. Talmud ${ }^{69}$, Fredrik

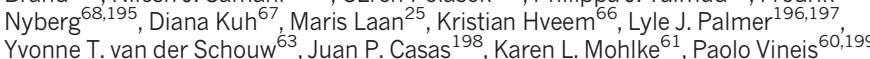

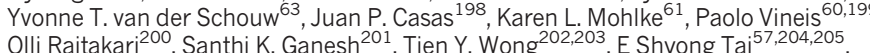
Richard S. Cooper ${ }^{54}$, Markku Laakso ${ }^{53}$, Dabeeru C. Rao ${ }^{206}$, Tamara B. Harris ${ }^{22}$, Richard W. Morris ${ }^{207}$, Anna F. Dominiczak ${ }^{208}$, Mika Kivimaki ${ }^{209}$, Michael G. Marmot $^{209}$, Tetsuro Miki ${ }^{49}$, Danish Saleheen ${ }^{48,169}$, Giriraj R. Chandak ${ }^{47}$, Josef Coresh $^{210}$, Gerjan Navis ${ }^{211}$, Veikko Salomaa ${ }^{125}$, Bok-Ghee Han ${ }^{44}$, Xiaofeng Zhu ${ }^{93}$, Jaspal S. Kooner ${ }^{41,159}$, Olle Melander ${ }^{39}$, Paul M' Ridker ${ }^{8,9,212}$, Stefania Bandinelli ${ }^{213}$, Ulf B. Gyllensten ${ }^{37}$, Alan F. Wright ${ }^{0}$, James F. Wilson ${ }^{34}$, Luigi Ferrucci ${ }^{33}$, Martin Farrall $^{32}$, Jaakko Tuomilehto $214,215,216,217$, Peter P. Pramstaller ${ }^{30,218}$, Roberto Elosua $^{29,219}$, Nicole Soranzo ${ }^{28,119}$, Eric J. G. Sijbrands ${ }^{13,14}$, David Altshuler ${ }^{114,220}$ Ruth J. F. Loos ${ }^{23}$, Alan R. Shuldiner ${ }^{26,221}$, Christian Gieger ${ }^{156}$, Pierre Meneton 222 ,' Andre G. Uitterlinden ${ }^{13,14,15}$, Nicholas J. Wareham ${ }^{23}$, Vilmundur Gudnason ${ }^{10,11}$, Jerome I. Rotter ${ }^{160}$, Rainer Rettig ${ }^{223}$, Manuela Uda ${ }^{174}$, David P. Strachan ${ }^{50}$, Jacqueline C. M. Witteman ${ }^{13,15}$, Anna-Liisa Hartikainen ${ }^{224}$, Jacques S. Beckmann ${ }^{104,225}$, Eric Boerwinkle 226 , Ramachandran S. Vasan 6,227 , Michael Boehnke ${ }^{31}$, Martin G. Larson ${ }^{6,228}$, Marjo-Riitta Järvelin 18,229,230,231,232, Bruce M. Psaty ${ }^{21,134 *}$, Gonçalo R. Abecasis ${ }^{19 *}$, Aravinda Chakravarti ${ }^{1 *}$, Paul Elliott ${ }^{18,232 *}$, Cornelia M. van Duijn ${ }^{13,233 *}$ Christopher Newton-Cheh ${ }^{27,114 *}$, Daniel Levy ${ }^{6,7,16 *}$, Mark J. Caulfield ${ }^{4 *}$ \& Toby Johnson ${ }^{4 *}$

${ }^{1}$ Center for Complex Disease Genomics, McKusick-Nathans Institute of Genetic Medicine, Johns Hopkins University School of Medicine, Baltimore, Maryland 21205, USA. ${ }^{2}$ Institute of Social and Preventive Medicine (IUMSP), Centre Hospitalier Universitaire Vaudois and University of Lausanne, Bugnon 17, 1005 Lausanne, Switzerland. ${ }^{3}$ Cardiology, Department of Specialties of Internal Medicine, Geneva University Hospital, Rue Gabrielle-Perret-Gentil 4, 1211 Geneva 14, Switzerland. ${ }^{4}$ Clinical Pharmacology and The Genome Centre, William Harvey Research Institute, Barts and The London School of Medicine and Dentistry, Queen Mary University of London, London EC1M 6BQ, UK. ${ }^{5}$ Department of Biostatistics, University of Washington, Seattle, Washington 98195, USA. ${ }^{6}$ Framingham Heart Study, Framingham, Massachusetts 01702, USA. ${ }^{7}$ National Heart Lung, and Blood Institute, Bethesda, Maryland 20824, USA. ${ }^{8}$ Division of Preventive Medicine, Brigham and Women's Hospital, 900 Commonwealth Avenue East, Boston, Massachusetts 02215, USA. ${ }^{9}$ Harvard Medical School, Boston, Massachusetts 02115 , USA. ${ }^{10}$ Icelandic Heart Association, 201 Kópavogur, Iceland. ${ }^{11}$ University of Iceland, 101 Reykajvik, Iceland. ${ }^{12}$ Department of Health Sciences, University of Leicester, University Rd, Leicester LE1 7RH, UK. ${ }^{13}$ Department of Epidemiology, Erasmus Medical Center, PO Box 2040, 3000 CA Rotterdam, The Netherlands. ${ }^{14}$ Department of Internal Medicine, Erasmus Medical Center, 3000 CA Rotterdam, The Netherlands. ${ }^{15}$ Netherlands Consortium for Healthy Aging (NCHA), Netherland Genome Initiative (NGI), Erasmus 3000 CA Rotterdam, The Netherlands. ${ }^{16}$ Center for Population Studies, National Heart Lung, and Blood Institute, Bethesda, Maryland 20824, USA. ${ }^{17}$ Department of Internal Medicine, Centre Hospitalier Universitaire Vaudois, 1011 Lausanne, Switzerland.

${ }^{18}$ Department of Epidemiology and Biostatistics, School of Public Health, Imperial College London, Norfolk Place, London W2 1PG, UK. ${ }^{19}$ Center for Statistical Genetics, Department of Biostatistics, University of Michigan School of Public Health, Ann Arbor, Michigan 48103, USA. ${ }^{20}$ Interfaculty Institute for Genetics and Functional Genomics, Ernst-Moritz-Arndt-University Greifswald, 17487 Greifswald, Germany. ${ }^{21}$ Cardiovascular Health Research Unit, Departments of Medicine, Epidemiology and Health Services, University of Washington, Seattle, Washington 98101, USA. ${ }^{22}$ Laboratory of Epidemiology, Demography, Biometry, National Institute on Aging, National Institutes of Health, Bethesda, Maryland 20892, USA. ${ }^{23}$ MRC Epidemiology Unit, Institute of Metabolic Science, Cambridge CB2 OQQ, UK. ${ }^{24}$ Centre National de Génotypage, Commissariat à L'Energie Atomique, Institut de Génomique, 91057 Evry, France. ${ }^{25}$ Institute of Molecular and Cell Biology, University of Tartu, Riia 23, Tartu 51010, Estonia. ${ }^{26}$ University of Maryland School of Medicine, Baltimore, Maryland 21201, USA. ${ }^{27}$ Center for Human Genetic Research, Cardiovascular Research Center, Massachusetts General Hospital, Boston, Massachusetts 02114, USA. ${ }^{28}$ Department of Twin Research \& Genetic Epidemiology, King's College London, London SE1 7EH, UK. ${ }^{29}$ Cardiovascular Epidemiology and Genetics, Institut Municipal d'Investigacio Medica, Barcelona Biomedical Research Park, 88 Doctor Aiguader, 08003 Barcelona, Spain. ${ }^{30}$ Institute of Genetic Medicine, European Academy Bozen/Bolzano (EURAC), Viale Druso 1, 39100 Bolzano, Italy - Affiliated Institute of the University of Lübeck, Germany. ${ }^{31}$ Department of Biostatistics, Center for Statistical Genetics, University of Michigan, Ann Arbor, Michigan 48109, USA. ${ }^{32}$ Department of Cardiovascular Medicine, The Wellcome Trust Centre for Human Genetics, University of Oxford, Oxford OX3 7BN, UK. ${ }^{33}$ Clinical Research Branch, National Institute on Aging, Baltimore, Maryland 21250, USA. ${ }^{34}$ Centre for Population Health Sciences, University of Edinburgh, EH8 9AG, UK. ${ }^{35}$ Centre for Population Health Sciences and Institute of Genetics and Molecular Medicine, College of Medicine and Vet Medicine, University of Edinburgh, EH8 9AG, UK. ${ }^{36}$ Croatian Centre for Global Health, 
University of Split, 21000 Split, Croatia. ${ }^{37}$ Department of Genetics and Pathology, Rudbeck Laboratory, Uppsala University, SE-751 85 Uppsala, Sweden. ${ }^{38}$ Amgen, 1 Kendall Square, Building 100, Cambridge, Massachusetts 02139, USA. ${ }^{39}$ Department of Clinical Sciences, Lund University, 20502 Malmö, Sweden. ${ }^{40}$ Department of Medicine, University of Verona, 37134 Verona, Italy. ${ }^{41}$ Ealing Hospital, London UB1 3HJ, UK. ${ }^{42}$ Department of Medicine, University of Mississippi Medical Center, Jackson, Mississippi 39216, USA. ${ }^{43}$ Genetic Epidemiology Group, Epidemiology and Public Health, UCL, London, WC1E 6BT, UK. ${ }^{44}$ Center for Genome Science, National Institute of Health, Seou 122-701, Korea. ${ }^{4}$ Department of Cardiology, University Medical Center Groningen, University of Groningen, $9713 \mathrm{GZ}$ Groningen, The Netherlands. ${ }^{46}$ Departments of Epidemiology and Medicine, Johns Hopkins University, Baltimore, Maryland 21205, USA. ${ }^{47}$ Centre for Cellular and Molecular Biology (CCMB), Council of Scientific and Industria Research (CSIR), Uppal Road, Hyderabad 500 007, India. ${ }^{48}$ Department of Public Health and Primary Care, University of Cambridge, CB1 8RN, UK. ${ }^{49}$ Department of Basic Medical Research and Education, and Department of Geriatric Medicine, Ehime University Graduate School of Medicine, Toon, 791-0295, Japan. ${ }^{50}$ Division of Community Health Sciences, St George's University of London, London SW17 ORE, UK. ${ }^{51}$ Epidemiology \& Prevention, Division of Public Health Sciences, Wake Forest University School of Medicine, Winston-Salem, North Carolina 27157, USA. ${ }^{52}$ Division of Biostatistics and Department of Genetics, School of Medicine, Washington University in St. Louis, Saint Louis, Missour 63110 , USA. ${ }^{53}$ Department of Medicine, University of Eastern Finland and Kuopio University Hospital, 70210 Kuopio, Finland. ${ }^{54}$ Department of Preventive Medicine and Epidemiology, Loyola University Medical School, Maywood, Illinois 60153, USA. ${ }^{55}$ Department of Laboratory Medicine \& Institute of Human Genetics, University of California San Francisco, 513 Parnassus Ave. San Francisco, California 94143, USA. ${ }^{56}$ Genome Institute of Singapore, Agency for Science, Technology and Research, Singapore 138672, Singapore. ${ }^{57}$ Centre for Molecular Epidemiology, Yong Loo Lin School of Medicine, National University of Singapore, Singapore 117597, Singapore. ${ }^{58}$ Department of Clinical Chemistry, University of Tampere and Tampere University Hospital, Tampere 33521, Finland. ${ }^{59}$ Department of Genetics, Biology and Biochemistry, University of Torino, Via Santena 19, 10126 Torino, Italy. ${ }^{60}$ Human Genetics Foundation (HUGEF), Via Nizza 52, 10126 Torino, Italy. ${ }^{61}$ Department of Genetics, University of North Carolina, Chapel Hill, North Carolina 27599, USA. ${ }^{62}$ MRC Centre for Causal Analyses in Translational Epidemiology, School of Social \& Community Medicine, University of Bristol, Bristol BS8 2BN, UK. ${ }^{63}$ Julius Center for Health Sciences and Primary Care, University Medical Center Utrecht, Heidelberglaan 100,3508 GA Utrecht, The Netherlands.

${ }^{64}$ Complex Genetics Section, Department of Medical Genetics - DBG, University Medica Center Utrecht, 3508 GA Utrecht, The Netherlands. ${ }^{65}$ Centre for Genetic Epidemiology and Biostatistics, University of Western Australia, Crawley, Western Australia 6009, Australia. ${ }^{66} \mathrm{HUNT}$ Research Centre, Department of Public Health and General Practice, Norwegian University of Science and Technology, 7600 Levanger, Norway. ${ }^{67}$ MRC Unit for Lifelong Health \& Ageing, London WC1B 5JU, UK. ${ }^{68}$ Occupational and Environmental Medicine, Department of Public Health and Community Medicine, Institute of Medicine, Sahlgrenska Academy, University of Gothenburg, 40530 Gothenburg, Sweden. ${ }^{69}$ Centre for Cardiovascular Genetics, University College London, London WC1E 6JF, UK. ${ }^{70} \mathrm{MRC}$ Human Genetics Unit and Institute of Genetics and Molecular Medicine, Edinburgh EH2, UK. ${ }^{71}$ Department of Cardiovascular Sciences, University of Leicester, Glenfield Hospital, Leicester LE3 9QP, UK. ${ }^{72}$ Leicester NIHR Biomedical Research Unit in Cardiovascular Disease, Glenfield Hospital, Leicester, LE3 9QP, UK. ${ }^{73}$ Studies Coordinating Centre, Division of Hypertension and Cardiac Rehabilitation, Department of Cardiovascular Diseases, University of Leuven, Campus Sint Rafaël, Kapucijnenvoer 35, Block D, Box 7001,3000 Leuven, Belgium. ${ }^{74}$ Center for Research on Genomics and Global Health, National Human Genome Research Institute, Bethesda, Maryland 20892, USA

${ }^{75}$ Columbia University, New York, New York 10027, USA. ${ }^{76}$ Department of Medicine III, Medical Faculty Carl Gustav Carus at the Technical University of Dresden, 01307 Dresden, Germany. ${ }^{77}$ Epidemiology and Biostatistics, School of Public Health, Imperial College, London W2 1 PG, UK. ${ }^{78}$ Clinical and Molecular Epidemiology Unit, Department of Hygiene and Epidemiology, University of loannina School of Medicine, 45110 loannina, Greece. ${ }^{79}$ Wake Forest University Health Sciences, Winston-Salem, North Carolina 27157, USA. ${ }^{80}$ Department of Epidemiology, School of Public Health, University of Michigan, Ann Arbor, Michigan 48109, USA. ${ }^{81}$ Division of Epidemiology, Human Genetics and Environmental Sciences, School of Public Health, University of Texas at Houston Health Science Center, 12 Herman Pressler, Suite 453E, Houston, Texas 77030, USA.

${ }^{82}$ Laboratory of Neurogenetics, National Institute on Aging, Bethesda, Maryland 20892. USA. ${ }^{8}$ Laboratory of Cardiovascular Science, Intramural Research Program, National Institute on Aging, $\mathrm{NIH}$, Baltimore, Maryland 21224, USA. ${ }^{84}$ Washington Hospital Center Division of Cardiology, Washington, District of Columbia 20010, USA. ${ }^{85}$ ALSPAC Laboratory, University of Bristol, Bristol BS8 2BN, UK. ${ }^{86}$ Pediatric Epidemiology Center, University of South Florida, Tampa, Florida 33612, USA. ${ }^{87}$ Clinical Pharmacology Unit, University of Cambridge, Addenbrookes Hospital, Hills Road, Cambridge CB2 2QQ, UK. ${ }^{88}$ University of Dundee, Ninewells Hospital \&Medical School, Dundee DD1 9SY, UK. ${ }^{89}$ Genetic Epidemiology Group, Department of Epidemiology and Public Health, UCL, London WC1E 6BT, UK. ${ }^{90}$ Pathology and Laboratory Medicine, University of Western Australia, Crawley, Western Australia 6009, Australia. ${ }^{91}$ Molecular Genetics, PathWest Laboratory Medicine, Nedlands, Western Australia 6009, Australia. ${ }^{92}$ Clinical Trial Service Unit and Epidemiological Studies Unit, University of Oxford, Oxford OX3 7LF, UK.

${ }^{93}$ Department of Epidemiology and Biostatistics, Case Western Reserve University, 2103 Cornell Road, Cleveland, Ohio 44106, USA. ${ }^{94}$ Department of Medicine, Johns Hopkins University, Baltimore 21205, USA. ${ }^{95}$ Department of Clinical Physiology, University of Tampere and Tampere University Hospital, Tampere, 33521, Finland. ${ }^{96}$ Department of Medicine, University of Turku and Turku University Hospital, Turku 20521, Finland. ${ }^{97}$ Department of Nutrition, University of North Carolina, Chapel Hill, North Carolina 27599, USA. ${ }^{98}$ Office of Population Studies Foundation, University of San Carlos, Talamban, Cebu City 6000, Philippines. ${ }^{99}$ Department of Neurology and Framingham Heart Study, Boston University School of Medicine, Boston, Massachusetts 02118, USA ${ }^{100}$ Department of Internal Medicine II, University Medical Center Regensburg, 93053 Regensburg, Germany. ${ }^{101}$ Department of Epidemiology and Preventive Medicine,
University Medical Center Regensburg, 93053 Regensburg, Germany. ${ }^{102}$ Department of Epidemiology, Johns Hopkins University, Baltimore, Maryland 21205, USA. ${ }^{103}$ Renal Division, University Hospital Freiburg, 79095 Freiburg, Germany. ${ }^{104}$ Département de Génétique Médicale, Université de Lausanne, 1015 Lausanne, Switzerland. ${ }^{105}$ Swiss Institute of Bioinformatics, 1015 Lausanne, Switzerland. ${ }^{106}$ Division of Genetics, GlaxoSmithKline, Philadelphia, Pennsylvania 19101, USA. ${ }^{107}$ International Centre for Circulatory Health, National Heart \& Lung Institute, Imperial College, London SW7 2AZ UK ${ }^{108}$ Genetics of Complex Traits, Peninsula Medical School, University of Exeter, Exeter EX4 4QJ, UK. ${ }^{109}$ Department of Community Health Sciences \& Department of Medicine, Aga Khan University, Karachi 74800, Pakistan. ${ }^{110}$ Medizinische Klinik II, Universität zu Lübeck, 23538 Lübeck, Germany. ${ }^{111}$ Diabetes Unit, KEM Hospital and Research Centre, Rasta Peth, Pune-411011, Maharashtra, India. ${ }^{112}$ Department of Clinical Sciences, Diabetes and Endocrinology Research Unit, University Hospital, 20502 Malmö, Sweden. ${ }^{113}$ Lund University, Malmö 20502, Sweden. ${ }^{114}$ Program in Medical and Population Genetics, Broad Institute of Harvard and MIT, Cambridge, Massachusetts 02139, USA. ${ }^{115}$ Department of Chronic Disease Prevention, National Institute for Health and Welfare, 00251 Helsinki, Finland. ${ }^{116}$ FIMM, Institute for Molecular Medicine, Finland, Biomedicum, P.O. Box 104, 00251 Helsinki, Finland. ${ }^{117}$ William Harvey Research Institute, Barts and The London School of Medicine and Dentistry, Queen Mary University of London, London EC1M 6BO, UK. ${ }^{118}$ Merck Research Laboratory, 126 East Lincoln Avenue, Rahway, New Jersey 07065, USA. ${ }^{119}$ Wellcome Trust Sanger Institute, Hinxton, CB10 1SA, UK. ${ }^{120}$ University of Cambridge Metabolic Research Labs, Institute of Metabolic Science Addenbrooke's Hospital, Cambridge CB2 OQQ, UK. ${ }^{121}$ Division of Cardiovascular Medicine, Department of Internal Medicine, University of Michigan Medical School, Ann Arbor, Michigan 48109, USA. ${ }^{122}$ Cardiovascular Genetics, University of Utah School of Medicine, Salt Lake City, Utah 84132, USA. ${ }^{123}$ Department of Physiology and Biophysics, Keck School of Medicine, University of Southern California, Los Angeles, California 90033, USA. ${ }^{124}$ National Human Genome Research Institute, National Institutes of Health, Bethesda, Maryland 20892,USA. ${ }^{125}$ National Institute for Health and Welfare, 00271 Helsinki, Finland. ${ }^{126}$ Broad Institute, Cambridge, Massachusetts 02142, USA.

${ }^{127}$ Leibniz-Institute for Arteriosclerosis Research, Department of Molecular Genetics of Cardiovascular Disease, University of Münster, 48149 Münster, Germany. ${ }^{128}$ Medical Faculty of the Westfalian Wilhelms University Muenster, Department of Molecular Genetics of Cardiovascular Disease, University of Münster, 48149 Münster, Germany. ${ }^{129}$ Division of Cardiology, Massachusetts General Hospital, Boston, Massachusetts 02114, USA. ${ }^{130}$ Georgia Prevention Institute, Department of Pediatrics, Medical College of Georgia, Augusta, Georgia 30912, USA. ${ }^{131}$ Unit of Genetic Epidemiology and Bioinformatics, Department of Epidemiology, University Medical Center Groningen, University of Groningen, $9713 \mathrm{GZ}$ Groningen, The Netherlands. ${ }^{132}$ Department of Biostatical Sciences, Division of Public Health Sciences, Wake Forest University School of Medicine, Winston-Salem, North Carolina 27157, USA. ${ }^{133}$ Department of Epidemiology, University of Washington, Seattle, Washington 98195, USA. ${ }^{134}$ Group Health Research Institute, Group Health Cooperative, Seattle, Washington 98124, USA. ${ }^{135}$ Seattle Epidemiologic Research and Information Center, Veterans Health Administration Office of Research \& Development, Seattle, Washington 98108, USA. ${ }^{136}$ Department of Medicine, University of Washington, Seattle, Washington 98195, USA. ${ }^{137}$ Department of Cardiology, University of Tartu, L. Puusepa 8, 51014 Tartu, Estonia. ${ }^{138}$ Tallinn University of Technology, Institute of Biomedical Engineering, Ehitajate tee 5, 19086 Tallinn, Estonia. ${ }^{139}$ Centre of Cardiology, North Estonia Medical Centre, Sütiste tee 19, 13419 Tallinn, Estonia. ${ }^{140}$ Department of Non-communicable disease Epidemiology, The London School of Hygiene and Tropical Medicine London, Keppel Street, London WC1E 7HT, UK ${ }^{141}$ South Asia Network for Chronic Disease, Public Health Foundation of India, C-1/52, SDA, New Delhi 100016, India. ${ }^{142}$ Department of Emergency and Cardiovascular Medicine, Institute of Medicine, Sahlgrenska Academy, University of Gothenburg, 41685 Gothenburg, Sweden. ${ }^{143}$ Department of Biostatistics, Institute of Basic Medical Sciences, University of Oslo, 0317 Oslo, Norway. ${ }^{144}$ Tuscany Regional Health Agency, 50129 Florence, Italy. ${ }^{145}$ Tropical Medicine Research Institute, University of the West Indies, Mona, Kingston, Jamaica. ${ }^{146}$ University of Ibadan, 200284 Ibadan, Nigeria.

${ }^{147}$ Department of Genomic Medicine, and Department of Preventive Cardiology, National Cerebral and Cardiovascular Research Center, Suita, 565-8565, Japan. ${ }^{148}$ Department of Health Science, Shiga University of Medical Science, Otsu, 520-2192, Japan.

${ }^{149}$ Department of Geriatric Medicine, Osaka University Graduate School of Medicine, Suita, 565-0871, Japan. ${ }^{150}$ Tohoku University Graduate School of Pharmaceutical Sciences and Medicine, Sendai, 980-8578, Japan. ${ }^{151}$ Lifestyle-related Disease Prevention Center, Shiga University of Medical Science, Otsu, 520-2192, Japan. ${ }^{152}$ Department of Medical Science and Cardiorenal Medicine, Yokohama City University School of Medicine, Yokohama, 236-0004, Japan. ${ }^{153}$ Department of Statistics, Pontificia Universidad Catolica de Chile, Vicuña Mackena 4860, Santiago, Chile. ${ }^{154}$ Institute of Human Genetics, Helmholtz Zentrum Munich, German Research Centre for Environmental Health, 85764 Neuherberg, Germany. ${ }^{155}$ Institute of Human Genetics, Klinikum rechts der Isar, Technical University of Munich, 81675 Munich, Germany. ${ }^{156}$ Institute of Epidemiology, Helmholtz Zentrum Munich, German Research Centre for Environmental Health, 85764 Neuherberg, Germany. ${ }^{157}$ Chair of Epidemiology, Institute of Medical Informatics, Biometry and Epidemiology, Ludwig-Maximilians-Universität, 81377 Munich, Germany. ${ }^{158}$ Klinikum Grosshadern, 81377 Munich, Germany. ${ }^{159}$ National Heart and Lung Institute, Imperial College London, London W12 OHS, UK. ${ }^{160}$ Medical Genetics Institute, Cedars-Sinai Medical Center, Los Angeles, California 90048, USA. ${ }^{161}$ Medical Population Genetics, Broad Institute of Harvard and MIT, 5 Cambridge Center, Cambridge, Massachusetts 02142, USA. ${ }^{162}$ National Heart, Lung and Blood Institute and its Framingham Heart Study, 73 Mount Wayte Ave., Suite \#2, Framingham, Massachusetts 01702, USA. ${ }^{163}$ Department of Neurology and Medicine, University of Washington, Seattle, Washington 98195, USA. ${ }^{164}$ Department of Medicine (Geriatrics), University of Mississippi Medical Center, Jackson, Mississippi 39216, USA. ${ }^{165}$ Department of Neurology, Boston University School of Medicine, Massachusetts 02118, USA. ${ }^{166}$ Finnish Institute of Occupational Health, Aapistie 1, 90220 Oulu, Finland. ${ }^{167}$ Wellcome Trust Centre for Human Genetics, University of Oxford, Oxford OX3 7BN, UK. ${ }^{168}$ Lapland Central Hospital, Department of Physiatrics, Box 8041, 96101 Rovaniemi, Finland. ${ }^{169}$ Center for 
Non-Communicable Diseases Karachi 74800, Pakistan ${ }^{170}$ Atherosclerosis Research Unit, Department of Medicine, Karolinska Institute, 17177 Stockholm, Sweden. ${ }^{171}$ Department of Internal Medicine, University Medical Center Groningen, University of Groningen, 9713 GZ Groningen, The Netherlands. ${ }^{172}$ Department of Clinical Genetics, Erasmus Medical Center, 3000 CA Rotterdam, The Netherlands. ${ }^{173}$ Gerontology Research Center, National Institute on Aging, Baltimore, Maryland 21224, USA. ${ }^{174}{ }^{4}$ stituto di Neurogenetica e Neurofarmacologia, Consiglio Nazionale delle Ricerche, Cittadella Universitaria di Monserrato, 09042 Monserrato, Cagliari, Italy. ${ }^{175}$ Unita Operativa Semplice Cardiologia, Divisione di Medicina, Presidio Ospedaliero Santa Barbara, 09016 Iglesias, Italy. ${ }^{176} \mathrm{Computational} \mathrm{Medicine} \mathrm{Research} \mathrm{Group,} \mathrm{Institute} \mathrm{of} \mathrm{Clinical} \mathrm{Medicine}$ University of Oulu and Biocenter Oulu, 90014 University of Oulu, Oulu, Finland. ${ }^{177}$ NMR Metabonomics Laboratory, Department of Biosciences, University of Eastern Finland, 70211 Kuopio, Finland. ${ }^{178}$ Department of Internal Medicine and Biocenter Oulu, Clinical Research Center, 90014 University of Oulu, Oulu, Finland. ${ }^{179}$ Institute for Molecular Medicine Finland FIMM, 00014 University of Helsinki, Helsinki, Finland. ${ }^{180}$ Department of Biomedical Engineering and Computational Science, School of Science and Technology, Aalto University, 00076 Aalto, Espoo, Finland. ${ }^{181}$ NUS Graduate School for Integrative Sciences \& Engineering (NGS) Centre for Life Sciences (CeLS), Singapore 117456, Singapore. ${ }^{182}$ Department of Internal Medicine B, Ernst-Moritz-Arndt-University Greifswald, 17487 Greifswald, Germany. ${ }^{183}$ Institute of Pharmacology, Ernst-Moritz-Arndt-University Greifswald, 17487 Greifswald, Germany. ${ }^{184}$ Institute for Community Medicine, Ernst-Moritz-Arndt-University Greifswald, 17487 Greifswald, Germany. ${ }^{185}$ U557 Institut National de la Santé et de la Recherche Médicale, U1125 Institut National de la Recherche Agronomique, Université Paris 13, 93017 Bobigny, France. ${ }^{186}$ Department of Neurology, Mayo Clinic, Jacksonville, Florida 32224, USA. ${ }^{187}$ Imperial College Cerebrovascular Unit (ICCRU), Imperial College, London W6 8RF, UK. ${ }^{188}$ Faculty of Medicine, University of Split, 21000 Split, Croatia. ${ }^{189}$ Department of Internal Medicine, Diabetology, and Nephrology, Medical University of Silesia, 41-800, Zabrze, Poland. ${ }^{190}$ Public Health Sciences section, Division of Community Health Sciences, University of Edinburgh, Medical School, Teviot Place, Edinburgh, EH89AG, UK. ${ }^{191}$ School of Science and Engineering, University of Ballarat, 3353 Ballarat, Australia. ${ }^{192}$ Prevention and Care of Diabetes, Department of Medicine III, Medical Faculty Carl Gustav Carus at the Technical University of Dresden, 01307 Dresden, Germany. ${ }^{193}$ University Hospital Münster, Internal Medicine D, 48149 Münster, Germany. ${ }^{194}$ Department of Medical Statistics, Epidemiology and Medical Informatics, Andrija Stampar School of Public Health, University of Zagreb, 10000 Zagreb, Croatia. ${ }^{195}$ AstraZeneca R\&D, 43183 MöIndal, Sweden. ${ }^{196}$ Genetic Epidemiology \& Biostatistics Platform, Ontario Institute for Cancer Research, Toronto, Ontario M5G 1L7, Canada. ${ }^{197}$ Samuel Lunenfeld Institute for Medical Research, University of Toronto, Toronto, Ontario ?M5S 1A1, Canada. ${ }^{198}$ Faculty of Epidemiology and Population Health, London School of Hygiene and Tropical Medicine London WC1E 7HT, UK. ${ }^{199}$ Department of Epidemiology and Public Health, Imperia College, Norfolk Place, London W2 1PG, UK. ${ }^{200}$ Research Centre of Applied and Preventive Cardiovascular Medicine, University of Turku and the Department of Clinical Physiology, Turku University Hospital, Turku, 20521, Finland. ${ }^{201}$ Department of Internal
Medicine, Division of Cardiovascular Medicine, University of Michigan Medical Center Ann Arbor, Michigan 48109, USA. ${ }^{202}$ Singapore Eye Research Institute, Singapore 168751 , Singapore. ${ }^{203}$ Department of Ophthalmology, National University of Singapore, Singapore 119074, Singapore. ${ }^{204}$ Department of Medicine, Yong Loo Lin School of Medicine, National University of Singapore, Singapore 119074, Singapore.

${ }^{205}$ Duke-National University of Singapore Graduate Medical School, Singapore 169857 Singapore. ${ }^{206}$ Division of Biostatistics, Washington University School of Medicine, Saint Louis, Missouri 63110, USA. ${ }^{207}$ Department of Primary Care \& Population Health, UCL, London NW3 2PF, UK. ${ }^{208}$ BHF Glasgow Cardiovascular Research Centre, University of Glasgow, 126 University Place, Glasgow G12 8TA, UK. ${ }^{209}$ Epidemiology Public Health, UCL, London WC1E 6BT, UK. ${ }^{210}$ Departments of Epidemiology, Biostatistics, and Medicine, Johns Hopkins University, Baltimore, Maryland 21205, USA. ${ }^{211}$ Division of Nephrology, Department of Internal Medicine, University Medical Center Groningen, University of Groningen, 9713 GZ Groningen, The Netherlands. ${ }^{212}$ Division of Cardiology, Brigham and Women's Hospital, 900 Commonwealth Avenue East, Boston, Massachusetts 02215, USA. ${ }^{213}$ Geriatric Rehabilitation Unit, Azienda Sanitaria Firenze (ASF), 50100 Florence, Italy. ${ }^{214}$ National Institute for Health and Welfare, Diabetes Prevention Unit, 00271 Helsinki, Finland. ${ }^{215} \mathrm{Hjelt}$ Institute, Department of Public Health, University of Helsinki, 00014 Helsinki, Finland. ${ }^{216}$ South Ostrobothnia Central Hospital, 60220 Seinäjoki, Finland. ${ }^{217}$ Red RECAVA Grupo RD06/0014/0015, Hospital Universitario La Paz, 28046 Madrid, Spain. ${ }^{218}$ Department of Neurology, General Central Hospital, 39100 Bolzano, Italy. ${ }^{219}$ CIBER Epidemiología y Salud Pública, 08003 Barcelona, Spain. ${ }^{220}$ Department of Medicine and Department of Genetics, Harvard Medical School, Boston, Massachusetts 02115, USA. ${ }^{221}$ Geriatric Research and Education Clinical Center, Veterans Administration Medical Center, Baltimore, Maryland 21201, USA. ${ }^{222}$ U872 Institut National de la Santé et de la Recherche Médicale, Centre de Recherche des Cordeliers, 75006 Paris, France. ${ }^{223}$ Institute of Physiology, Ernst-Moritz-Arndt-University Greifswald, 17487 Greifswald, Germany. ${ }^{224}$ Institute of Clinical Medicine/Obstetrics and Gynecology, University of Oulu, 90014 Oulu, Finland. ${ }^{225}$ Service of Medical Genetics, Centre Hospitalier Universitaire Vaudois, 1011 Lausanne Switzerland. ${ }^{226}$ Human Genetics Center, 1200 Hermann Pressler, Suite E447 Houston, Texas 77030, USA. ${ }^{227}$ Division of Epidemiology and Prevention, Boston University Schoo of Medicine, Boston, Massachusetts 02215. USA ${ }^{228}$ Department of Mathematics, Boston University, Boston, Massachusetts 02215, USA. ${ }^{229}$ Institute of Health Sciences, University of Oulu, BOX 5000, 90014 University of Oulu, Finland. ${ }^{230}$ Biocenter Oulu, University of Oulu, BOX 5000, 90014 University of Oulu, Finland. ${ }^{231}$ National Institute for Health and Welfare, Box 310, 90101 Oulu, Finland. ${ }^{232}$ MRC-HPA Centre for Environment and Health, School of Public Health, Imperial College London, Norfolk Place, London W2 1PG, UK. ${ }^{233}$ Centre of Medical Systems Biology (CMSB 1-2), NGI Erasmus Medical Center, Rotterdam, The Netherlands.

*These authors contributed equally to this work.

$\uparrow$ A full list of authors and affiliations appears in Supplementary Information. tDeceased. 